\title{
Fire Data Analysis and Feature Reduction Using Computational Intelligence Methods
}

Majid Bahrepour, Berend Jan van der Zwaag, Nirvana Meratnia, and Paul Havinga

\begin{abstract}
Fire is basically the fast oxidation of a substance that produces gases and chemical productions. These chemical productions can be read by sensors to yield an insight about type and place of the fire. However, as fires may occur in indoor or outdoor areas, the type of gases and therefore sensor readings become different. Recently, wireless sensor networks (WSNs) have been used for environmental monitoring and real-time event detection because of their low implementation costs and their capability of distributed sensing and processing. In this paper, the authors investigate spatial analysis of data for indoor and outdoor fires using data-mining approaches for WSN-based fire detection purposes. This paper also delves into correlated data features in fire data sets and investigates the most contributing features for fire detection applications.
\end{abstract}

\section{Introduction}

Fire may take place in various places and may be caused by different mechanisms. Its humanitarian, economical and environmental catastrophic effects vary depending on its type (causing factor), location, scale, etc. Despite all the precautions, the US National Interagency Fire Center reports occurrence of 15,918 fires affecting 403,509 acres in 2009 alone [15]. Fast and effective fire detection is the key in fighting the fire. To this end, over the years various technologies and detection mechanisms have been developed and used not only to speed up the detection process but also to enhance reliability of the information analysis. The fact that fire is basically the fast oxidation of a substance that produces gases and chemical productions makes high spatial and temporal resolution sensing technologies such as wireless sensor networks (WSNs) a better candidate for fast fire detection compared to often-used low spatial and temporal resolution remote sensing and satellite im-

Majid Bahrepour · Berend Jan van der Zwaag · Nirvana Meratnia · Paul Havinga

University of Twente, The Netherlands, e-mail: \{m.bahrepour,b.j.vanderzwaag,n.meratnia,p.j.m.havinga\}@utwente.nl 


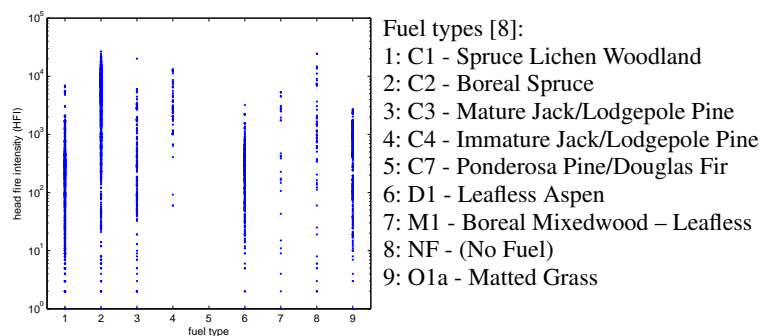

a Fuel type vs HFI

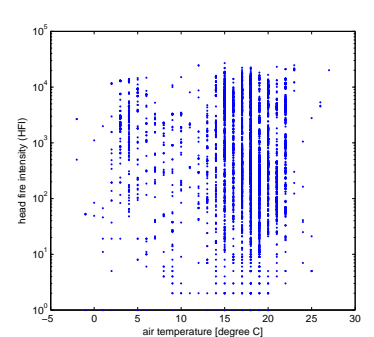

b Temperature vs HFI

Fig. 1 Scatter plots of fuel type and ambient temperature versus head fire intensity (HFI)

agery. Wireless sensor networks consist of a large number of tiny, usually batterypowered devices equipped with radio, memory, processing units as well as different type of sensors (e.g., temperature, humidity, smoke) being densely deployed in an environment to monitor a phenomenon.

A careful analysis of existing research in the field of fire detection reveals that while much attention has been paid to developing complex detection algorithms, not equal attention has been paid to analysis of fire data to identify the most effective features and the optimal set of features (sensors). In this paper, by applying data analysis and feature extraction techniques originated from computational intelligent field on two fire data sets, i.e., wildfire and residential fire, we identify the most contributing features (sensors) to the process of fire detection [1,2]. We then apply our distributed naive Bayes and neural network outlier detection techniques designed for WSNs on the extracted features and present the performance evaluation.

\section{Wildfire Data Analysis}

Some of the input features in a data set are more important than others, with respect to how well they predict an output feature. In other words, the less important features do not effect the predictability of the system significantly. Therefore, in order to reduce computational effort, we want to reduce the number of features that are used for predicting the system's output. There are many methods that accomplish such a feature reduction, and we will illustrate one here, based on iteratively reducing the number of features until an optimal set of features is found.

\subsection{Data Set}

The data set we have used contains a wide range of features used by Canadian forest fire management information systems [8]. Features used in these systems comprise 
geographical data (latitude, longitude, province), timing data (date, time), environmental data (ecoregion, ecozone, fuel type), weather data (air temperature, relative humidity, wind speed and direction, precipitation), sensor information (sensor type, platform) and sets of codes and indices used by two fire management information systems: the Canadian forest fire weather index system FWI (fine fuel moisture code, duff moisture code, drought code, initial spread index, buildup index, fire weather index) and the Canadian forest fire behavior prediction system FBP (crown fraction burned, total fuel consumption, head fire rate of spread, head fire intensity). The relation between some features is shown in the scatter plots of Fig. 1.

\subsection{Feature Extraction Method}

For our experiments, we use an initial set of 15 features as input features (latitude (LA), longitude (LO), province (PV), date (DA), time (TI), ecoregion (ER), ecozone (EZ), fuel type (FT), air temperature (TE), relative humidity (RH), wind speed (WS), wind direction (WD), precipitation (PC), sensor type (ST) and platform (SP)) and one feature that we want to predict, namely head fire intensity (HFI).

In order to reduce the computational complexity of our prediction mechanism and because we expect that most features only marginally contribute to the predictive ability of our method, we reduce the number of features to an optimal set of features that yields the best prediction accuracy.

Our feature reduction method is iterative. In the first iteration, we determine the prediction accuracy using the full set of input features. In each following iteration, we determine which feature has the least contribution to the prediction accuracy and then remove this feature from the set of input features.

Because some input features seem to contradict each other when predicting the HFI, the prediction accuracy will actually increase when removing those contradicting features, until the reduced set of input features is so small that reducing it even further would cause the accuracy to decrease again. We call the feature set size optimal if removing any more features would significantly worsen the prediction accuracy.

\subsubsection{HFI Prediction}

We use a Kohonen self-organizing map [7] to predict the HFI in the following way. First, we split our data set randomly in a training set and a test set. As we have a large data set, a relatively small part is sufficient for training; our training set contains $20 \%$ (2458 samples) of the entire data set; the test set the remaining $80 \%$ (9835 samples).

Second, we train a self-organizing map with the training set for a fixed number of epochs $(20000)$. We found that this number, along with the lattice size $(20 \times 20)$ and other learning parameters give best results in preliminary experiments. Ideally, the 

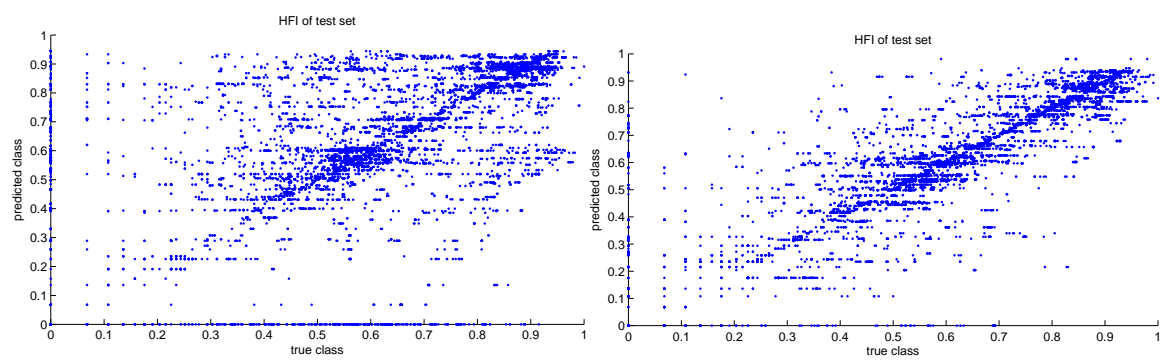

a For all features: $E_{\mathrm{c}}=0.334, E_{\mathrm{MSE}}=0.061$

b For four features: $E_{\mathrm{c}}=0.074, E_{\mathrm{MSE}}=0.013$

Fig. 2 Scatter plots of predicted HFI versus ground truth. Ideally, all points should lie on the line where $H F I_{\text {predicted }}=H F I_{\text {true }}$

self-organizing map will cluster data samples that are similar. After training, each node in the Kohonen map will contain a vector that is representative for the sample data vectors (in the training set).

Third, we assign labels to the nodes in the map. Each node is assigned the HFI of the data sample (in the training set) that is most similar (i.e., has the smallest Euclidean distance) to the vector stored in the node.

Fourth, we calculate an error measure by checking to which node in the Kohonen map each sample in the test set is mapped, and comparing the sample's true HFI with its predicted HFI, which is the HFI of the node in the map. Figure 2 shows two scatter plots of predicted HFI versus true HFI for the full set of input features and for an optimal set of four features, respectively.

For comparison, we have used two different error measures: $E_{\mathrm{c}}=1-c_{x y}$, where $c_{x y}$ is the correlation between all test samples' HFI values $x$ and the HFI values $y$ of the corresponding nodes in the Kohonen map; and $E_{\mathrm{MSE}}=\frac{1}{N} \sum(x-y)^{2}$, the mean squared error (MSE).

If the error is zero, the predictor is perfect. In practice this will not be the case, so we try to find the predictor that has the smallest error. In order to reduce the influence of statistical variations each experiment is repeated one hundred times with different random splits of the data set, and the final error measure per feature set is averaged accordingly.

\subsection{Results}

Our method successfully reduced the number of features to a representative set of features. Figure 3 a shows the progressive results using correlation as optimization criterion and Fig. 3b presents the results using MSE. In both cases the fuel type (FT) is the most significant feature, and together with date (DA), precipitation (PC), and ecozone (EZ) a comprehensive set of four features has been found that produces the best predictor for head fire intensity (HFI). 


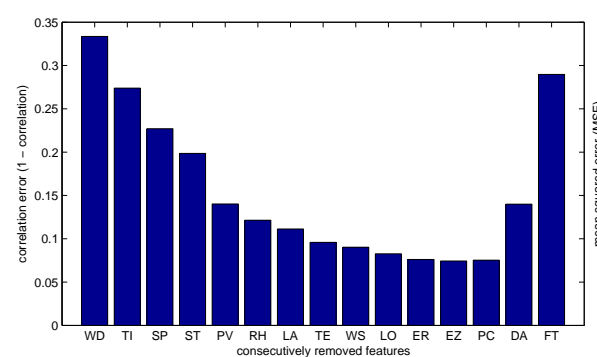

a Results using correlation

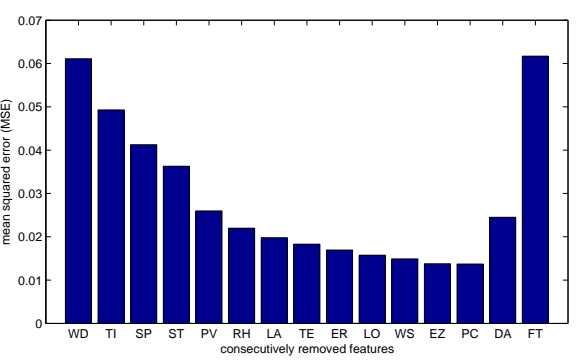

b Results using MSE

Fig. 3 Resulting error measures when progressively removing one feature at a time from the initial full feature set. Consecutively removed features are listed on the horizontal axis; the right-most bars show the error measure for a single feature, which in both cases is the fuel type

\section{Residential Fire Data Analysis}

By looking back to the basic notions on residential fire detection, it is seen that earlier works were conducted by only one sensor type [1]. Temperature, flame detector and air-obscuration sensors are mostly used as an individual sensor for residential fire detection $[1,2]$. However, investigation of gas sensors such as carbon monoxide (CO) demonstrated that gas sensors contribute the most to fire detection techniques $[1,13,14]$. Recent studies show that temperature, ionization, photoelectric, and CO sensors together make an optimal sensor set for residential fire detection [1, 2, 3]. Therefore, we use these four sensors to analyze residential fire data and further to detect residential fire. There is an invaluable database on residential fire provided by National Institute of Standards and Technology (NIST, http://www.nist.gov) from which we make a sample data set. Residential fires are categorized into flaming (having flames and making massive heats) and smoldering (having less flames and heats and more smokes) fires. Additionally, some actions such as toasting a bread or lighting a cigarette may generate nuisances that can be mistaken with real fire. Hence to have a global insight into residential fire data, before analyzing the data we first combine data sets of various flaming fires, smoldering fires and nuisances. Figure 4 shows a 2D plot of temperature, ionization, photoelectric and $\mathrm{CO}$ sensor data generated by residential fires.

From Fig. 4a, we can see that temperature sensor can be a fine indicator of flaming fires, as flaming fires produce massive heats. However, nuisances and smoldering fires are overlapping each other and the temperature sensor is not a good separator between smoldering fires and nuisances. Figure $4 \mathrm{~b}$ shows that although some overlaps exist between the three types of fire, ionization can distinguish well between smoldering fires and the other two. Figure $4 \mathrm{c}$ shows that the photoelectric sensor can make a distinction between the three classes to some extent, as smoldering fires have lower values, while nuisances have intermediate values and flaming fires have the highest values. Figure $4 \mathrm{~d}$ shows that $\mathrm{CO}$ separates nuisances well, as flaming fires and smoldering fires have a value around zero and nuisances have a va- 


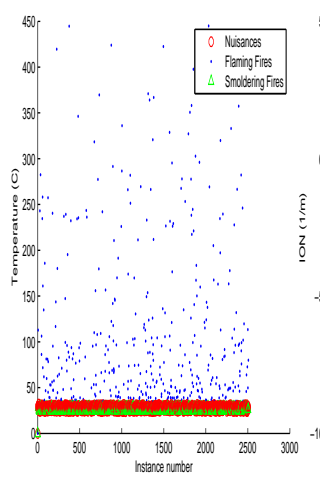

a Temperature

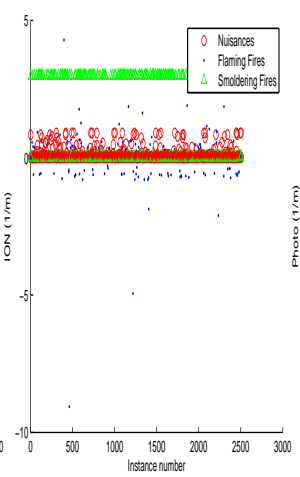

b Ionization

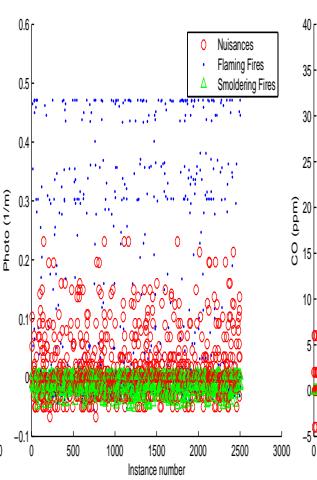

c Photoelectric

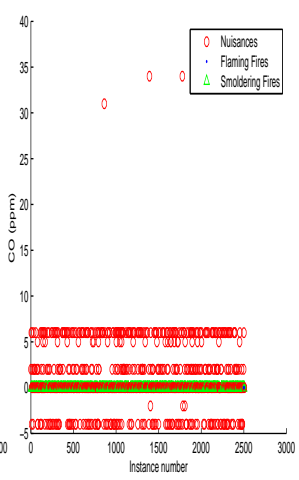

d Carbon monoxide

Fig. 4 Scatter plots for four features of residential fire data set \#2

riety of values. Table 1 presents the correlations between sensors. As it can be seen in this table, correlations are less than $10 \%$ in all situations except the correlation between $\mathrm{CO}$ and photo, which is almost $-20 \%$. Therefore, we can say that almost all features are independent.

Table 1 Correlation between features

\begin{tabular}{lcccc}
\hline & Temperature & Ionization & Photoelectric CO gas \\
\hline Temperature & 1 & -0.061 & 0.097 & -0.044 \\
Ionization & -0.061 & 1 & 0.087 & -0.036 \\
Photoelectric & 0.097 & 0.087 & 1 & -0.208 \\
CO gas & -0.044 & -0.036 & -0.208 & 1 \\
\hline
\end{tabular}

\subsection{Contribution of Features to the Fire Detection}

Finding the optimal set of features (sensors) to robustly and accurately detect fire has been investigated in several studies. As a result, having temperature, ionization, photo and $\mathrm{CO}$ sensors are proposed as the minimum set of sensors for fire detection $[1,2,3]$. Although the fire detection can be conducted with only one sensor, fire detection using all four sensors is more robust to environmental noise and provides extra support for sensor failures. The exact contribution of each sensor to the fire detection varies based on the classifier or detection algorithm. Nevertheless, it has been reported that $\mathrm{CO}$ is the most contributing feature $[1,2,3]$. In Table 2, we report detection accuracies of the 16 possible combinations of sensors using decision trees, neural networks, and naive Bayes classifiers. A brief introduction to neural networks, naive Bayes, and decision trees is presented next. 


\subsubsection{Neural Networks, Naive Bayes, and Decision Tree Classifiers}

An artificial neural network (ANN) is a computational model inspired by biological neural networks. It consists of an interconnected collection of artificial neurons and processes information using a connectionist method $[2,19]$. As a simple modeling of biological neural networks, feed-forward neural networks (FFNN) consist of one input layer, one or more hidden layers and one output layer. Inputs are the data and the output is a predicted value (class) that input data is expected to belong to. The Naive Bayes classifier uses Bayesian statistics and Bayes' theorem to find the probability of each instance belonging to a specific class. It is called naive because of its emphasis on independency of the input data [2]. Decision trees are decisionsupporting tools that represent a set of if-then-else rules as tree-like graphs. To make a decision using decision trees, variables are evaluated from the root of the tree down to the leaves. Finally, tree-node evaluations lead to a single leaf, which returns a value and is considered as the result of the decision-making process.

Table 2 Residential fire detection using decision tree, feed-forward neural network, and naive Bayes classifiers

\begin{tabular}{ccccccc}
\hline \multicolumn{3}{c}{ Combinations of sensor types used } & \multicolumn{3}{c}{$\begin{array}{c}\text { Detection accuracies for three classifiers } \\
\text { Temperature Ionization Photoelectric CO gas }\end{array}$} \\
\hline$\checkmark$ & $\checkmark$ & $\checkmark$ & $\checkmark$ & 99.08 & 98.69 & 71.58 \\
- & $\checkmark$ & $\checkmark$ & $\checkmark$ & 98.53 & 98.00 & 71.18 \\
$\checkmark$ & - & $\checkmark$ & $\checkmark$ & 96.78 & 97.43 & 70.27 \\
$\checkmark$ & $\checkmark$ & - & $\checkmark$ & 98.80 & 98.53 & 71.25 \\
$\checkmark$ & $\checkmark$ & $\checkmark$ & - & 89.25 & 70.00 & 63.00 \\
- & - & $\checkmark$ & $\checkmark$ & 95.52 & 97.30 & 70.20 \\
$\checkmark$ & - & - & $\checkmark$ & 95.58 & 95.00 & 71.78 \\
$\checkmark$ & $\checkmark$ & - & - & 63.78 & 97.00 & 60.05 \\
- & $\checkmark$ & $\checkmark$ & - & 61.28 & 68.73 & 62.80 \\
- & $\checkmark$ & - & $\checkmark$ & 98.17 & 98.00 & 71.13 \\
$\checkmark$ & - & $\checkmark$ & - & 80.67 & 66.80 & 63.28 \\
- & - & - & $\checkmark$ & 97.33 & 94.00 & 70.43 \\
- & - & $\checkmark$ & - & 52.43 & 84.18 & 61.05 \\
- & $\checkmark$ & - & - & 37.50 & 45.68 & 62.85 \\
$\checkmark$ & - & - & - & 44.83 & 84.48 & 62.50 \\
\hline
\end{tabular}

\subsection{Analysis of Fire Detection Techniques}

By looking back to the basic notions of fire alarms using electronic devices, it can be seen that smoke sensors are the preliminary tools for detecting fires. Smoke sensors are generally either responsive to air ionization or obscuration [5]. The problem with 
such simple detectors is that they are prone to false alarms because they assume that only fires and nothing else may produce smoke.

Generally speaking, the existing WSN-based fire detection techniques are either threshold-based $[10,16,17,18]$ or pattern-matching based $[6,9,12,21]$. Threshold based techniques define a threshold value for their sensor readings and when the sensor value is larger or smaller than the pre-defined threshold value, an alarm is generated. As, finding a predefined threshold value is not something straightforward, Liang and Wang presents an automatically-selected threshold value approach [10] in which a sliding window technique automatically finds the threshold values, dynamically. In case of having more than one feature, Vu et al. propose evaluating various sensor values separately by considering them as an "atom" or distinct value [17]. For example, if fire is detected using both smoke and temperature sensors, an alarm will be generated when temperature exceeds $30^{\circ} \mathrm{C}$ and smoke exceeds 100 $\mathrm{mg} / \mathrm{L}$. Lim et al. [11] introduce a generic fire detection and rescue support system, which they say is applicable for any other disaster recoveries.

In the pattern-matching studies, techniques such as contour maps [20], sensorreading maps [6], distributed fuzzy logic [12], neural networks, naive Bayes, and support vector machines $[1,2,3,4]$ have been proposed. Map-based studies define an acceptable range for sensor values, which exceeding from it generates an alarm indicating a fire event. In pattern matching studies, sample data are needed to train the classifier. Then, the classifier conducts the pattern matching either inside a node or by several nodes in a distributed fashion. Generally, as a new trend in fire or other event detection practices in WSNs the pattern-matching techniques are proposed in a distributed manner $[1,2,3,4,12]$.

\section{Distributed Wildfire and Residential Fire Detection}

As mentioned earlier, the new trend in WSN fire detection is to use a distributed approach to perform the detection by using several nodes. Therefore, we use the distributed approach presented in [3] to provide reports on the residential and wildfire detection. In the following subsections the approach and results are described.

\subsection{The Distributed Approach Using Data Fusion}

A notion of detecting events inside each sensor nodes (irrespective to the available sensor types in the sensor nodes) and then fuse the all results in a higher level is presented in [3]. Figure 5 shows the general topology of this approach.

Sensor nodes and the data fuser can use any classifier or algorithm to do its classification or fusion job. However, naive Bayes (NB) and feed forward neural network (FFNN) are already used for this approach. Here we also used NB and FFNN to see their accuracy on the analyzed residential and wildfire data sets. The 
Fig. 5 Block diagram of the approach presented in [3]

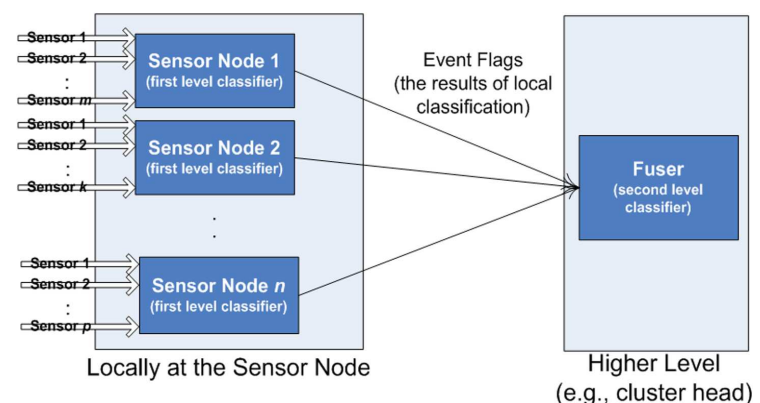

results are reported in Table 3 . The experiment method is the same as original study in [3].

Table 3 The results of residential fire and wildfire detection using the distributed approach in [3]

\begin{tabular}{lllll}
\hline & \multicolumn{4}{l}{ Distributed approach using FFNN } \\
& Mean & STD & Mean & STD \\
\hline Wildfire & 93.29 & 1.61 & 92.64 & 2.47 \\
Residential fire & 85.73 & 1.98 & 81.40 & 10.52 \\
\hline
\end{tabular}

\section{Conclusion}

This paper presents a thorough data analysis on wildfire and residential fire data sets using feature extraction and machine-learning techniques. The contribution of the paper is on providing an insight on finding the correlation between various features and identifying the most contributing features to the goal of fire detection. This identified set forms the optimal set of sensors that can efficiently and reliably detect fires.

Experimental results of applying our distributed neural network and naive Bayes based fire detection techniques on this optimal set prove high fire detection accuracy, i.e., over $81 \%$ for residential fire detection and over $92 \%$ for wildfire detection.

Acknowledgements This paper describes work in part undertaken in the context of the SENSEI project, "Integrating the Physical with the Digital World of the Network of the Future" (www.senseiproject.eu). SENSEI is a Large Scale Collaborative Project supported by the European 7th Framework Programme, contract number: 215923. 


\section{References}

1. Bahrepour, M., Meratnia, N., Havinga, P.J.M.: Automatic fire detection: a survey from wireless sensor network perspective. Technical Report TR-CTIT-08-73, Centre for Telematics and Information Technology, University of Twente, Enschede. ISSN 1381-3625 (2008)

2. Bahrepour, M., Meratnia, N., Havinga, P.J.M.: Use of AI techniques for residential fire detection in wireless sensor networks. In: AIAI 2009, Thessaloniki, Greece (2009)

3. Bahrepour, M., Meratnia, N., Havinga, P.J.M.: Sensor fusion-based event detection in wireless sensor networks. In: SensorFusion'09, Toronto, Canada. IEEE (2009)

4. Bahrepour, M., Zhang, Y., Meratnia, N., Havinga, P.J.M.: Use of event detection approaches for outlier detection in wireless sensor networks. In: ISSNIP 2009, Melbourne, Australia (2009)

5. Brain, M.: How smoke detectors work. Available from: http://home.howstuffworks.com /smoke1.htm (2000)

6. Jin, G., Nittel, S.: NED: an efficient noise-tolerant event and event boundary detection algorithm in wireless sensor networks. In: 7th International Conference on Mobile Data Management, IEEE Computer Society (2006)

7. Kohonen, T.: Self-organized formation of topologically correct feature maps. Biological Cybernetics 43, pp. 59-69 (1982)

8. Lee, B.S., Alexander, M.E., Hawkes, B.C., Lynham, T.J., Stocks, B.J., Englefield, P.: Information systems in support of wildland fire management decision making in Canada. Computers and Electronics in Agriculture 37, pp. 185-198, Elsevier (2002)

9. Li, D., Wong, K.D., Hu, Y.H., Sayeed, A.M.: Detection, classification, and tracking of targets. Signal Processing Magazine 19(2):17-29, IEEE (2002)

10. Liang, Q., Wang, L.: Event detection in sensor networks using fuzzy logic system,” In: EEE International Conference on Computational Intelligence for Homeland Security and Personal Safety, Orlando, FL, USA (2005)

11. Lim, Y.-s., Lim, S., Choi, J., Cho, S., Kim, C.-k., Lee, Y.-W.: A fire detection and rescue support framework with wireless sensor networks. In: International Conference on Convergence Information Technology, IEEE Computer Society, pp. 135-138 (2007)

12. Marin-Perianu, M., Havinga, P.: D-FLER - a distributed fuzzy logic engine for rule-based wireless sensor networks. Springer Lecture Notes in Computer Science 4836 (2008)

13. Milke, J.A.: Using multiple sensors for discriminating fire detection. In: Fire Suppression and Detection Research Application Symposium, National Fire Protection Research Foundation, pp. 150-164 (1999)

14. Milke, J.A., McAvoy, T.J.: Analysis of signature patterns for discriminating fire detection with multiple sensors. Fire Technology 31(2):120-136 (1995)

15. National Interagency Fire Center: Fire Information - National Fire News. Retreived from http://www.nifc.gov/fire_info/nfn.htm on 17 March 2010

16. Segal, M.L., Antonio, F.P., Elam, S., Erlenbach, J., de Paolo, K.R.: Method and apparatus for automatic event detection in a wireless communication system, USA Patent (2000)

17. Vu, C.T., Beyah, R.A., Li, Y.: Composite event detection in wireless sensor networks," In: Performance, Computing, and Communications Conference (IPCCC 2007), IEEE International (2007)

18. Werner-Allen, G., Lorincz, K., Ruiz, M., Marcillo, O., Johnson, J., Lees, J., Welsh, M.: Deploying a wireless sensor network on an active volcano. IEEE Internet Computing 10(2):1825 (2006)

19. Wikipedia: Artificial neural network. Http://en.wikipedia.org/wiki/Artificial_neural_network accessed in March 2010

20. Xue, W., Luo, Q., Chen, L., Liu, Y.: Contour map matching for event detection in sensor networks. In: International Conference on Management of Data. ACM New York, NY, USA (2006)

21. Zoumboulakis, M., Roussos, G.: Escalation: complex event detection in wireless sensor networks. Springer Lecture Notes in Computer Science 4793, pp. 270-285 (2007) 